\title{
Shing-Tung Yau and Sixty Years of Calabi's Conjecture
}

\section{by Kefeng Liu}

\author{
Department of Mathematics, University of California at Los Angeles
}

The 1950s belong to one of the most glorious decades for geometry and topology. A group of young mathematicians established a wealth of great theorems, made ground breaking discoveries, and created a new era in mathematics. These men leave their names in the history of mathematics.

Hodge theory was just completed by Weyl and Kodaira in 1941. By exploiting the Chern characteristic classes introduced by Shiing-Shen Chern, Hirzebruch proved the signature theorem and the Hirzebruch-Riemann-Roch theorem in algebraic geometry in 1956. The engineer-turn-mathematician Bott proved the periodicity theorem on homotopy groups. These results would soon inspire Atiyah and Singer to prove their index theorem. Serre calculated the homotopy groups of spheres in algebraic topology using Leray spectral sequence, wrote the famous GAGA in algebraic geometry using sheaf theory, and systematically introduced complex analysis to algebraic geometry. Kodaira proved his famous embedding theorem, and developed deformation theory of complex manifolds. Later, Milnor found the first exotic sphere in dimension 7 and Nash proved his isometric embedding theorem for Riemannian manifolds. These great results are like stars shining in the sky.

On Amsterdam's 1954 International Congress of Mathematicians, Kodaira and Serre were awarded Fields medals. Both of their works merged complex analysis, differential geometry and algebraic geometry in a miraculous fashion. As Weyl said in his famous encomium: "You reached heights of which I never dreamt... It shows that the old gnarled tree of mathematics is still full of sap and life."

On the very occasion, Eugenio Calabi, a 31-year-old Italian-American mathematician, wrote down his famous conjecture on a sheet of paper during his talk, "For any (1,1)-form $R$ in the first Chern class of a compact Kähler manifold, there exists a unique Kähler metric whose Ricci form is equal to $R$." Calabi established the uniqueness of the metric and outlined a program to solve his conjecture.

However, three years later, in his 1957 paper "On Kähler manifolds with vanishing canonical class," Calabi pointed that his approach would not work, for one needs to solve a very complicated nonlinear partial differential equation called the complex Monge-Ampère equation. He went to ask André Weil, one of the greatest mathematicians of the 20th century. Weil said: "Nowadays, mathematics is not ready to solve it."

As is well known, the famous Poincaré uniformization theorem tells us that there are only three universal coverings of one-dimensional complex manifolds, namely, the sphere, the complex plane and the unit disc. These correspond to 1-dimensional Kähler manifolds of constant positive, zero, and negative curvature, respectively.

Attempts to find suitable generalization of the uniformization theorems to higher dimensional manifolds have been an important development in topology, geometry and the theory of several complex variables. Even for the passage from one complex dimension to two complex dimensions, the difficulty of the problem has been far beyond one's imagination. Mathematicians like to joke that while God created the simple and beautiful Riemann surfaces, the devil gave us the complicated and bewildering complex surfaces.

Calabi's conjecture is an elegant, bold extension of the uniformization program to higher dimensions, pointing us to remarkably general phenomena that are otherwise not readily discernible among higher dimensional complex manifolds. In particular, when the first Chern class of a compact Kähler manifold is equal to or less than zero, it guarantees the existence of a Kähler manifold with constant zero, or negative Ricci curvature. These are the so-called Kähler-Einstein manifolds. This existence theorem therefore gives an indication of the complexity of the problem of generalizing the uniformization of Riemann surfaces to higher dimensions.

At that time, all known examples of Einstein manifolds were locally homogeneous, and people did not even know whether Kähler-Einstein metrics exist on complex hypersurfaces of projective spaces such as the K3 surfaces. It was under this circumstance that Calabi made his audacious speculation based on his profound insight. It is no wonder that the majority of geometers doubted the correctness of this conjecture. People were struggling to find a counter-example rather than prove it. 
Like Poincaré's uniformization theorem and Hodge's theorem whose complete solutions took decades, Calabi's conjecture, which is of fundamental importance in unveiling the global meaning of Ricci curvature for Kähler manifolds, had to wait 22 years for its proof.

In 1954, the year Calabi published his conjecture, Shing-Tung Yau was five year old and living in poverty on the other side of the world-Hong Kong. Several years later, the sudden death of his father dealt a big blow to the 14-year-old boy, but the drastic turn of fortune also helped shape his indomitable character. He entered the Chinese University of Hong Kong and, in 1969, he went

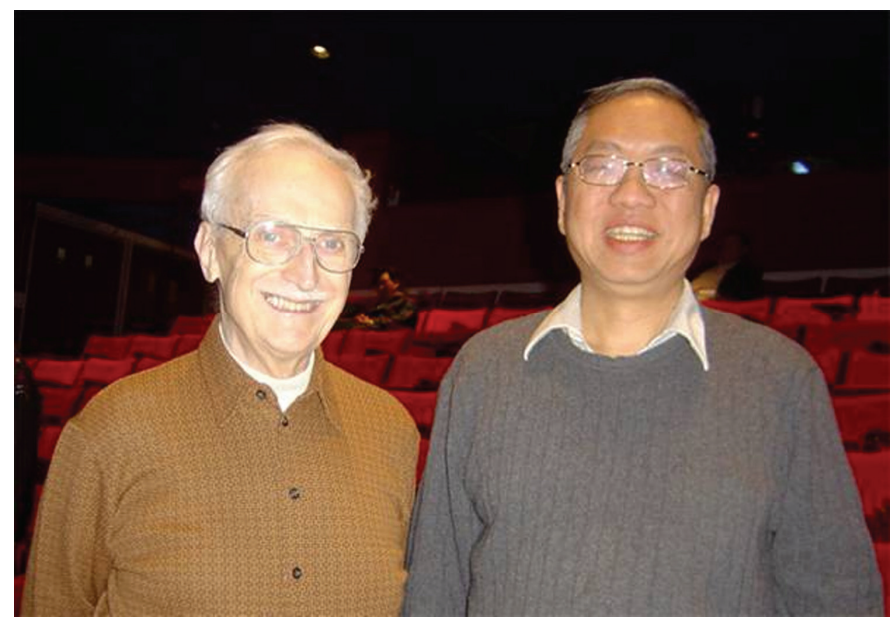

Eugenio Calabi and Shing-Tung Yau

abroad as a college senior to study at Berkeley. That year, Hung-Hsi Wu, a geometer at Berkeley, wrote to another geometer Robert Greene, predicting that the 19-year-old youngster will change the landscape of differential geometry. We do not know how Professor Wu saw the unusual talents in a 19-year-old young man.

During the first year of his postgraduate study, although he was still a newcomer to geometry, Shing-Tung Yau solved a certain problem on the structure of the fundamental group of manifolds with negative curvature. Only afterward did he learn that he had in fact solved a well-known conjecture of Professor Joe Wolf at Berkeley. It is similar to the legend that John Milnor solved a conjecture in knot theory after he had mistaken it to be an exercise.

Later, when he learned of the Calabi conjecture, like meeting a beautiful girl, he fell in love with it at first sight. The rest of the story is by now well known, but only Yau himself could feel the pain and joy behind the proof. Later he would attribute the secret of his success to hard work, not talent. He had to try out numerous test functions in order to develop the technique of gradient estimation on manifolds. To solve the Calabi's conjecture, it was necessary to create and develop nonlinear analysis on manifold systematically in order to handle the fully nonlinear Monge-Ampère equation.
Working with Shiu-Yuen Cheng, he solved the famous Minkowski problem and Bernstein problem in Minkowski space-time using the real Monge-Ampère equation. Then he pushed the technique of gradient estimates to the utmost and finally solved Calabi's conjecture in 1976. At that moment, another happy person in addition to Yau should be Calabi. From 1954 to 1976, Calabi had to wait twenty-two years before finally realizing his dream. In that year, Calabi, Yau and Nirenberg spent the whole Christmas day on discussing Yau's proof at the Courant Institute in New York city. Calabi's conjecture finally became the Calabi-Yau theorem.

Later Calabi recalled that it is the only time in his life to have a meeting on Christmas day and that Yau's proof was his best Christmas gift. When he received the Lifetime Achievement Award by the American Mathematical Society in 1991, he acknowledged Yau, whose work helped him receive the award.

Serre once said: "A really good mathematical conjecture is one whose resolution brings forth a series of corollaries and a long lasting influence." Calabi's conjecture is one such example. Let me enumerate a few striking consequences that follow from the solution of the Calabi conjecture.

On compact Kähler manifold with zero or negative first Chern class, Calabi's conjecture tells us that Kähler-Einstein metrics always exist. The negative case of this existence theorem leads to the proof of the long-standing Severi conjecture on the uniqueness of the complex structure of the complex projective plane. In fact,

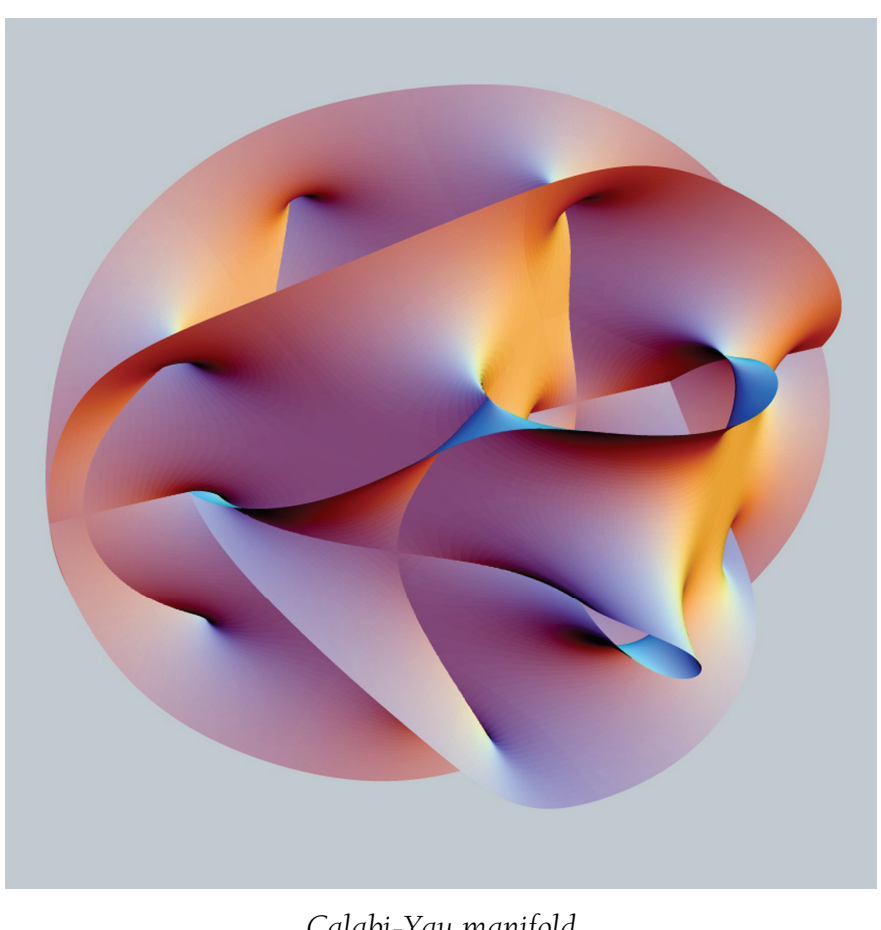

the complex Kähler structure of complex projective spaces in all dimensions is also unique. Another fantastic corollary is that on such complex manifolds, there is a 
marvelous inequality between Chern numbers. Before that, algebraic geometers could only obtain weaker inequalities for complex surfaces. This is the famous Bogomolov-Miyaoka-Yau inequality, which is an optimal inequality of Chern number for algebraic surfaces conjectured to be true by Van de Ven. It turns out that by making use of the Kähler-Einstein metric, Yau was able to understand what happens to the equality case. That $3 c_{2}=c_{1}^{2}$ implies such algebraic manifolds are quotients of the ball. Up to now, this is still the only proof of this remarkable fact that quotient of the ball is characterized by topological numbers only.

Two dimensional complex manifolds with zero first Chern class are the famous K3 surfaces. Siu and Todorov were able to use the Calabi-Yau metric to simplify the arguments of Kulikov on the proof of the subjectivity of the period map for K3 surfaces. Using the Calabi-Yau theorem, Siu proved that every K3 surface is Kähler.

The fundamental structural theorem for higher dimensional complex manifolds with zero first Chern class was also established using theorems in Riemannian geometry about manifolds of zero Ricci curvature.

All these results were famous conjectures in complex and algebraic geometry. The new analytic tool of the Kähler-Einstein metric has been the major tool to solve some of these old conjectures. In some cases, there were algebraic geometric proof, but often the Kähler-Einstein metric helps to simplify the arguments drastically. On one occasion, Manin offered the opinion that the Kähler-Einstein metric should play an important role in the transcendental part of Arakelov geometry.

In 1984, when string theory had its first breakthrough due to Green-Schwarz, they wanted to build a model to realize phenomenological question in string theory. Candelas-Horowitz-Strominger-Witten proposed to use Yau's Ricci flat Kähler manifolds to perform"compactification of string theory" to four dimensional effective theory. And they built a beautiful theory based on such manifolds. They called such manifolds to be Calabi-Yau manifolds.

By now, if one inputs "Calabi-Yau" in Google, one may find more than 400,000 entries. The Calabi-Yau space has led to a series of major breakthroughs in algebraic geometry and other related subjects. A very important contribution by the joint efforts of physicists and mathematciains was the work surrounding the concept of "mirror symmetry." The physicists that were involved include Candelas et al. Greene, Harvey, Klemm, Lüst, Moore, Plesser, Vafa, Witten and others, and mathematicians include Kontesvich, Givental, Lian, Liu, Yau, Batyrev, Fukaya, Gross, Hitchin, Hosono, Li, Manin, Morrison, Oh, Pandharipande, Ruan, Seidel, Siebert, Soibelman, Zaslow, Zinger and others. The mirror conjecture proved by Givental and Lian-Liu-Yau independently solved some century-old problems in enumerative geometry. The development of Witten's Chern-Simons theory is related to topological string theory on Calabi-Yau manifolds where beautiful formulas were conjectured by Vafa and his coauthors. These formulas were proved in my joint works with C.-C. Melissa Liu and J. Zhou.

The proof of Calabi's conjecture also marks a new era in transcendental algebraic geometry. Hodge theory, Kodaira's embedding theorem and the Calabi-Yau theorem are the three milestones in complex geometry. They have many features in common; they are all proved by using tools from differential geometry and are the essential bridge connecting geometry to other fields such as algebraic geometry. Assumptions in these theorems are simple and easy to verify, and they apply to a large class of manifolds. Moreover, they have led to a wide range of applications.

The proof of Calabi's conjecture also marks a new era in differential geometry. A new discipline called geometric analysis was born. In geometric analysis, methods of nonlinear differential equations are employed to solve problems in geometry and topology and, conversely, geometric ideas are used to understand partial differential equations.

Yau presented a clear overview of the prospects of geometric analysis and highdimensional uniformization theory in his plenary talk at the International Congress of Mathematicians in 1978. Strongly influenced by his approach, a series of well-known problems were settled. In particular, the application of gauge field theory and low-dimensional topology by Simon Donaldson and others as well as the study of the Ricci flow that led to the breakthrough in the three dimensional Poincaré conjecture by Richard Hamilton and Perelman brought the development of geometry analysis to its zenith.

As early as 1983, Huaidong Cao and Bando, under Yau's guidance, first used the method of Ricci flow to study the existence of canonical metrics on Kähler manifolds, making Kähler-Ricci flow one of the important tools in the study of complex manifolds.

Another problem closely related to Calabi's conjecture is the correspondence between stability and Hermitian-Einstein metrics on holomorphic vector bundles in algebraic geometry. This problem reduces to the solvability of some extremely difficult nonlinear equations arising from the gauge field theory. In 1986, Shing-Tung Yau, in collaboration with Uhlenbeck, completely solved the problem for the case of Kähler manifolds. Later, Donaldson solved the same problem by a different method for projective manifolds. In 1988, these results were generalized and developed (in combination with Hodge theory) into an extremely effective tool in algebraic geometry by Simpson.

On the tangent bundle of a complex manifold, a Kähler-Einstein metric can be regarded as a Hermitian-Einstein metric with no torsion. Existence of a Kähler-Einstein metric on the manifold means that the tangent bundle is stable in the sense of algebraic geometry, but the general case is more subtle. Over the years, 
Yau has been considering what kind of algebraic stability corresponds to the existence of Kähler-Einstein metrics.

In 1988 when I came to Harvard to study with Yau, most topics in his students' seminars were on various notions of stability in algebraic geometry and related topics. Doctoral theses of several students of Yau, including those of Gang Tian, Jun Li, Conan Leung and Huazhang Luo, addressed problems on this topic. Some of the ideas are recorded in the article " 100 geometric problems" in 1990, which was published in celebration of the 79th birthday of Prof. S. S. Chern. In Problem 65, it is conjectured that the existence of Kähler-Einstein metrics is equivalent to the stability in the sense of geometric invariant theory in algebraic geometry. On a complex manifold with positive first Chern class, this conjecture gives a necessary and sufficient condition of existence of Kähler-Einstein metrics for the first time. This conjecture establishes a close relationship between canonical metrics and algebraic geometry. Many of his students at that time, including Gang Tian, started to work in the new direction.

Prior to this, Yau also considered how to approximate Kähler-Einstein metrics via Bergman kernels and how to generalize Calabi's conjecture to open manifolds and manifolds with singularities. He discussed these issues in several influential review articles. They have become the framework for the future development of complex geometry, and inspired the works of Donaldson and Tian on Kähler-Einstein metrics. In 1980s, Yau collaborated with Cheng, Mok and Tian in a series of papers on Kähler-Einstein metrics, some of which formed the basis of Tian's doctoral dissertation.

In contrast with the case of non-positive first Chern class, the case of positive Chern class was quite obscure until Yau proposed his conjecture. There are examples of manifold with positive first Chern class and no Kähler-Einstein metrics. In the 1960s, Matsushima proved that the automorphism group of a Kähler-Einstein manifold must be reductive. In the early 1980s, Futaki found an obstruction on the existence of Kähler-Einstein metrics on these manifolds which is now called Futaki invariants. In fact, many scholars, such as Calabi and Futaki, incorrectly believed that the non-existence of holomorphic vector fields is the only necessary condition for the existence of Kähler-Einstein metrics. The importance of the stability of a manifold was not yet realized. In the special case of complex two-dimensional manifolds, there are some existence results in 1990s, which were pointed out to be incomplete by Siu: a convincing proof of the semi-continuity of singularity exponents was missing. This issue was eventually settled only some 10 years later.

In order to understand the relationship of stability and the existence of Kähler-Einstein metrics under positive curvature conditions, Tian defined a concept of analytic stability, so called K-stability, via Futaki invariants and made some progress in this direction. A real breakthrough came from Donaldson, who proved in 2001 that a Kähler manifold possessing a constant scalar curvature
Kähler metric and a discrete automorphism group is stable in the sense of algebraic geometry. The key tool used by Donaldson is the approximation method via the Bergman kernel, which was proposed by Yau in his review article based on his IMU Lectures at ETH Zurich in 1981. Donaldson proved that if the scalar curvature is a constant then an associated partial differential equation is solvable by using the asymptotic expansion of the Bergman kernel whose second term is exactly the scalar curvature, a fact first observed by Z. Lu. Later Donaldson introduced another concept of K-stability in the sense of algebraic geometry, which is suitable for the study of Yau's conjecture. In 2010, Donaldson proposed a program to solve Yau's conjecture on the equivalence between K-stability and existence of Kähler-Einstein metrics. The program has been completed recently by three long papers posted on the arXiv.org preprint server authored by X. Chen, S. Donaldson and S. Sun.

Complex manifolds with positive first Chern class, also called Fano manifolds, are relatively scarce compared

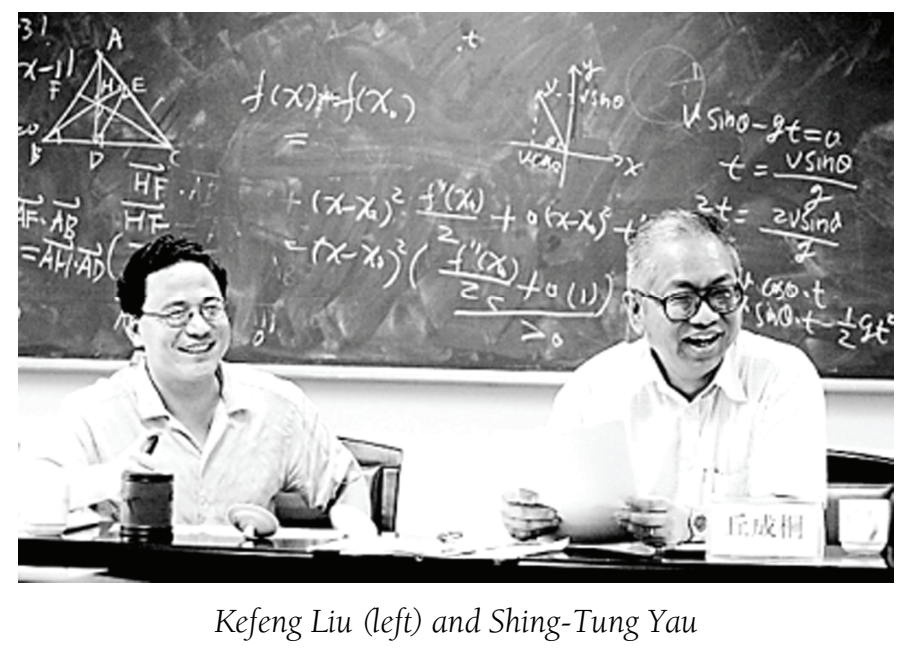

with manifolds with nonpositive first Chern class.

For example, a complex one dimensional Fano manifold is a sphere and a complex two dimensional Fano manifold is the complex projective plane with some points blown up. For Fano manifolds, it seems that analytic methods based on the Kähler-Einstein metric is not as powerful as those provided by algebraic methods.

In fact, in 1979 Mori proved the existence of rational curves on Fano manifolds by using algebro-geometric techniques in positive characteristic. Differential geometric methods so far have not been able to go beyond this ingenuous method. In contrast, the situation is quite different for manifolds with first Chern class less than or equal to zero. They admit far more examples than Fano manifolds. Since Yau's proof of Calabi's conjecture, differential geometry provides more powerful and effective methods and tools in the study of these manifolds.

Up to now, Yau's idea to use Kähler Einstein metric to study algebraic manifold with nonpositive first Chern class has been very fruitful. It is clear that it will continue 
to flourish. On the other hand, while it is not so clear that Yau's conjecture will provide similar powerful tool for Fano manifolds, Yau pointed out that an importance consequence of his conjecture is that Donaldson's $\mathrm{K}$-stable Fano manifolds will have stable tangent bundle in the sense of Mumford. This is a remarkable fact that is not clear for algebraic geometers.

The recent interest of Yau has moved toward recent developments in string theory where he found many new challenges and inspirations.

Calabi's conjecture is only part of Yau's mathematical achievements. He was invited to make one-hour plenary address at the international congress of mathematicians in 1978 at the age 29. He was 34 when he received the Fields medal, the greatest honor for young mathematicians, in 1983. It is worthwhile to note that he was holding a Hong Kong passport at that time as a citizen of China, a fact which he has always been proud of. In December 22, 1983, the General Secretary of CPC Central Committee, Mr. Yaobang $\mathrm{Hu}$ had a meeting with Yau in Zhongnanhai. Since then, he has received most of the highest honors a mathematician can get, including the Wolf Prize, the Crafoord Prize, and the U.S. National Medal of Science. The proof of Calabi's conjecture is without a doubt the most brilliant chapter in his mathematics career. This proof launched a brilliant era of geometric analysis in the last 40 years. 